\title{
LANGUAGE CLASSES ASSOCIATED WITH AUTOMATA OVER MATRIX GROUPS
}

\author{
Özlem Salehi ${ }^{(A)} \quad$ Flavio D'Alessandro ${ }^{(B, C)}$ \\ Ahmet Celal Cem Say ${ }^{(A)}$ \\ ${ }^{(A)}$ Boğaziçi University, Department of Computer Engineering, \\ Bebek 34342 İstanbul, Turkey \\ \{ozlem.salehi, say\}@boun.edu.tr \\ ${ }^{(B)}$ Boğaziçi University, Department of Mathematics, \\ Bebek 34342, İstanbul, Turkey \\ ${ }^{(C)}$ Università di Roma "La Sapienza", Dipartimento di Matematica, \\ Piazzale Aldo Moro 2, 00185 Roma, Italy \\ dalessan@mat.uniroma1. it
}

\begin{abstract}
We investigate the language classes recognized by group automata over matrix groups. We present a summary of the results obtained so far together with a number of new results. We look at the computational power of time-bounded group automata where the group under consideration has polynomial growth.
\end{abstract}

\section{Introduction}

Many extensions of the classical finite automaton model have been examined. One such variant is the group automaton (finite automaton over groups), which is a nondeterministic finite automaton equipped with a register which holds an element from a group. The register is initialized to the identity element of the group, and a computation is deemed successful if the register is equal to the identity element at the end of the computation after being multiplied at every step. This setup generalizes various models such as nondeterministic blind multicounter automata [8], and finite automata with multiplication [11].

Group automata were defined explicitly for the first time in the paper [16]. The theory of group automata has been essentially developed in the case of free groups [4, 5, 13, and in the case of free Abelian groups [6,7], where strong theorems allow to characterize the power of such

(A) Özlem Salehi is partially supported by TÜBİTAK (Scientific and Technological Research Council of Turkey).

${ }^{(B)}$ Flavio D'Alessandro acknowledges the support of 7th FP TÜBİTAK/Marie-Curie Co-Funded Brain Circulation Scheme, 2236. 
models and the combinatorial properties of the languages recognized by these automata. The connection between the word problem of a group and the class of languages recognized by the associated automaton has been essential while analysing group automata. Other papers which deal with group automata and the word problems of groups include [3, 12].

Our aim in this paper is to provide an overview of the languages recognized by finite automata over matrix groups. Even in the case of groups of matrices of low dimension that are not of the type mentioned above, the study of group automata becomes quickly nontrivial, and there are remarkable classes of linear groups for which little is known about the automaton models that they define. We present some new results about the classes of languages recognized by finite automata over matrix groups. We also introduce the notion of time complexity for group automata and use this to prove an impossibility result about word problems of groups with exponential growth.

Section 2 contains definitions and introduces notation that will be used throughout the paper. In Section 3, we focus on matrix groups with integer and rational entries. For the case of $2 \times 2$ matrices, we prove that the corresponding group automata for rational matrix groups are more powerful than the corresponding group automata for integer matrix groups. We explore finite automata over some special matrix groups such as the discrete Heisenberg group and the Baumslag Solitar group. In Section 4, we consider group automata operating in bounded time and prove that the word problem of a group with exponential growth cannot be recognized by a finite automaton over a group with polynomial growth in polynomial time, whereas in the succeeding section we summarize various results from the literature and some additional new related results. Section 6 lists some open questions.

\section{Preliminaries}

\subsection{Notation and Terminology}

The following notation will be used throughout the paper: $Q$ is the set of states, $q_{0} \in Q$ denotes the initial state, $Q_{a} \subseteq Q$ denotes the set of accepting states, and $\Sigma$ is the input alphabet.

An input string $w$ is placed between two endmarker symbols on an infinite tape in the form c $w \$$. By $w^{r}$, we represent the reverse of the string $w$. The length of $w$ is denoted by $|w|$.

REG, CF, and RE denote the family of regular languages, context-free languages, and recursively enumerable languages, respectively.

We assume a familiarity with some basic notions from algebra and group theory (see [9], [15] for references on this topic). For a finitely generated group $G$ and a set $A$ of generators, the word problem language of $G$ is the language $W(G, A)$ over $A$ which consists of all words that represent the identity element of $G$. Most of the time, the statements about word problem are independent of the generating set and in these cases the word problem language is denoted by $W(G)$. 


\subsection{Group Automaton}

Group automata first appear explicitly in the paper The accepting power of finite automata over groups by Mitrana and Stiebe [16] under the name of extended finite automaton. The definition is formally given as follows.

Let $K=(M, \circ, e)$ be a group under the operation denoted by $\circ$ with the neutral element denoted by $e$. An extended finite automaton over the group $K=(M, \circ, e)$ is a 6 -tuple

$$
\mathcal{E}=\left(Q, \Sigma, K, \delta, q_{0}, Q_{a}\right)
$$

where the transition function $\delta$ is defined as

$$
\delta: Q \times(\Sigma \cup\{\varepsilon\}) \rightarrow \mathbb{P}(Q \times M) .
$$

$\delta(q, \sigma) \ni\left(q^{\prime}, m\right)$ means that when $\mathcal{E}$ reads the symbol (or empty string) $\sigma \in \Sigma \cup\{\varepsilon\}$ in state $q$, it will move to state $q^{\prime}$, and write $x \circ m$ in the register, where $x$ is the old content of the register. The initial value of the register is the neutral element $e$ of the group $K$. The string is accepted if, after completely reading the string, $\mathcal{E}$ enters an accept state with the content of the register being equal to the neutral element of $K$.

We will prefer using the name group automaton ( $G$-automaton) instead of extended finite automaton over group $G$.

The class of languages recognized by $G$-automaton will be denoted as $\mathfrak{L}(G)$.

\section{Matrix Groups and Associated Language Classes}

In this section, we are going to prove some new results about the classes of languages recognized by finite automata over various groups, focusing on linear groups.

We will denote the free group over $r$ generators by $\mathbf{F}_{r}$. Note that $\mathbf{F}_{0}$ is the trivial group, and $\mathbf{F}_{1}$ is isomorphic to $\mathbb{Z}$, the additive group of integers. The class of regular languages is characterized as the set of languages recognized by finite automata over the trivial group $\mathbf{F}_{0}$ in $[5]$.

We will denote by $\mathbb{Z}^{k}$ the additive group of integer vectors of dimension $k$. This group is isomorphic to the free Abelian group of rank $k$, and $\mathbb{Z}^{k}$-automata are equivalent to nondeterministic blind $k$-counter automata $[10]$. We denote by $\mathbb{Q}^{+}$the multiplicative group of positive rational numbers, which is isomorphic to a free Abelian group of infinite rank. A $\mathbb{Q}^{+}$-automaton is also equivalent to a nondeterministic finite automaton with multiplication without equality (1NFAMW) of Ibarra et al. [11.

A characterization of context-free languages by group automata was first stated by Dassow and Mitrana [5], and proven in [4]. Let us note that $\mathbf{F}_{2}$ contains any free group of rank $n \geq 2$ [15]. 
Fact $3.14,5,12, \mathfrak{L}\left(\mathbf{F}_{2}\right)$ is the family of context-free languages.

We denote by $G L(2, \mathbb{Z})$ the general linear group of degree two over the field of integers, that is the group of $2 \times 2$ invertible matrices with integer entries. Note that these matrices have determinant \pm 1 . Restricting the matrices in $G L(2, \mathbb{Z})$ to those that have determinant 1 , we obtain the special linear group of degree two over the field of integers, $S L(2, \mathbb{Z})$.

Let $\mathbf{G}$ be the group generated by the matrices

$$
M_{a}=\left[\begin{array}{ll}
1 & 2 \\
0 & 1
\end{array}\right] \quad \text { and } \quad M_{b}=\left[\begin{array}{ll}
1 & 0 \\
2 & 1
\end{array}\right] .
$$

There exists an isomorphism $\varphi$ from $\mathbf{F}_{2}$ onto $\mathbf{G}$ by [14]. Note that $M_{a}$ and $M_{b}$ are integer matrices with determinant 1 , which proves that $\mathbf{F}_{2}$ is a subgroup of $S L(2, \mathbb{Z})$.

Now the question is whether $\mathfrak{L}(G L(2, \mathbb{Z}))$ and $\mathfrak{L}(S L(2, \mathbb{Z}))$ correspond to larger classes of languages than the class of context-free languages. We are going to use the following fact to prove that the answer is negative.

Fact 3.2 [4] Suppose $G$ is a finitely generated group and $H$ is a subgroup of finite index. Then $\mathfrak{L}(G)=\mathfrak{L}(H)$.

Now we are ready to state our theorem.

Theorem $3.3 \mathfrak{L}(S L(2, \mathbb{Z}))=\mathfrak{L}(G L(2, \mathbb{Z}))=$ CF.

Proof. We are going to use Fact 3.2 to prove the result. Since $S L(2, \mathbb{Z})$ has index 2 in $G L(2, \mathbb{Z})$ and $G L(2, \mathbb{Z})$ is finitely generated, $\mathfrak{L}(G L(2, \mathbb{Z}))=\mathfrak{L}(S L(2, \mathbb{Z}))$. Since $\mathbf{F}_{2}$ has index 12 in $S L(2, \mathbb{Z})[2]$ and $S L(2, \mathbb{Z})$ is finitely generated, $\mathfrak{L}(S L(2, \mathbb{Z}))=\mathfrak{L}\left(\mathbf{F}_{2}\right)$ which is equal to the family of context-free languages by Fact 3.1 .

Let us now investigate the group $S L(3, \mathbb{Z})$, the group of $3 \times 3$ integer matrices with determinant 1.

We start by looking at an important subgroup of $S L(3, \mathbb{Z})$, the discrete Heisenberg group. The discrete Heisenberg group $\mathbf{H}$ is defined as $\langle a, b \mid a b=b a c, a c=c a, b c=c b\rangle$ where $c=a^{-1} b^{-1} a b$ is the commutator of $a$ and $b$.

$$
a=\left[\begin{array}{lll}
1 & 1 & 0 \\
0 & 1 & 0 \\
0 & 0 & 1
\end{array}\right] \quad b=\left[\begin{array}{lll}
1 & 0 & 0 \\
0 & 1 & 1 \\
0 & 0 & 1
\end{array}\right] \quad c=\left[\begin{array}{lll}
1 & 0 & 1 \\
0 & 1 & 0 \\
0 & 0 & 1
\end{array}\right]
$$

Any element $g \in \mathbf{H}$ can be written uniquely as $b^{j} a^{i} c^{k}$.

$$
g=\left[\begin{array}{lll}
1 & i & k \\
0 & 1 & j \\
0 & 0 & 1
\end{array}\right]=b^{j} a^{i} c^{k}
$$


It is shown in [18] that the languages MULT $=\left\{x^{p} y^{q} z^{p q} \mid p, q \geq 0\right\}, \operatorname{COMPOSITE}=\left\{x^{p q} \mid p, q>\right.$ $1\}$ and MULTIPLE $=\left\{x^{p} y^{p n} \mid p \in \mathbb{N}\right\}$ can be recognized by a $\mathbf{H}$-automaton, using the special multiplication property of the group.

Correcting a small error in [18], we rewrite the multiplication property of the elements of $\mathbf{H}$.

$$
\left(b^{x} a^{y} c^{z}\right)\left(b^{x^{\prime}} a^{y^{\prime}} c^{z^{\prime}}\right)=b^{x+x^{\prime}} a^{y+y^{\prime}} c^{z+z^{\prime}+y x^{\prime}}
$$

We can make the following observation using the fact that $\mathfrak{L}(\mathbf{H})$ contains non-context-free languages.

Theorem $3.4 \mathfrak{L}(S L(2, \mathbb{Z})) \subsetneq \mathfrak{L}(S L(3, \mathbb{Z}))$.

Proof. It is obvious that a $S L(2, \mathbb{Z})$-automaton can be simulated by a $S L(3, \mathbb{Z})$-automaton. Note that $\mathfrak{L}(S L(2, \mathbb{Z}))$ is the family of context-free languages by Theorem 3.3 . Since $\mathfrak{L}(\mathbf{H}) \subsetneq$ $\mathfrak{L}(S L(3, \mathbb{Z}))$ and the non-context-free language COMPOSITE $=\left\{x^{p q} \mid p, q>1\right\}$ can be recognized by a $\mathbf{H}$-automaton [18], the result follows.

Now let us move on to the discussion about matrix groups with rational entries.

Let us denote by $G L(2, \mathbb{Q})$ the general linear group of degree two over the field of rational numbers, that is, the group of invertible matrices with rational entries. Restricting the matrices in $G L(2, \mathbb{Q})$ to those that have determinant 1 , we obtain the special linear group of degree two over the field of rationals, $S L(2, \mathbb{Q})$.

We will start by proving that allowing rational entries enlarges the class of languages recognized by matrices with determinant 1 .

Theorem $3.5 \mathfrak{L}(S L(2, \mathbb{Z})) \subsetneq \mathfrak{L}(S L(2, \mathbb{Q}))$.

Proof. It is obvious that $\mathfrak{L}(S L(2, \mathbb{Z})) \subseteq \mathfrak{L}(S L(2, \mathbb{Q}))$. We will prove that the inclusion is proper.

Let us construct a $S L(2, \mathbb{Q})$-automaton $\mathcal{G}$ recognizing the language $\mathrm{L}=\left\{a^{2^{2 n+1}} \mid n \geq 0\right\}$. The state diagram of $\mathcal{G}$ and the matrices are given in Figure1. Without scanning any input symbol, $\mathcal{G}$ first multiplies its register with the matrix $A_{1} \cdot \mathcal{G}$ then multiplies its register with the matrix $A_{2}$ successively until nondeterministically moving to the next state. After that point, $\mathcal{G}$ starts reading the string and multiplies its register with the matrix $A_{3}$ for each scanned $a$. At some point, $\mathcal{G}$ nondeterministically stops reading the rest of the string and multiplies its register with the matrix $A_{4}$. After sucessive multiplications with $A_{4}, \mathcal{G}$ nondeterministically decides moving to an accept state.

Let us trace the value of the register at different stages of the computation. Before reading the first input symbol, the register has the value

$$
\left[\begin{array}{cc}
2^{x+1} & 0 \\
2^{x} & \frac{1}{2^{x+1}}
\end{array}\right]
$$




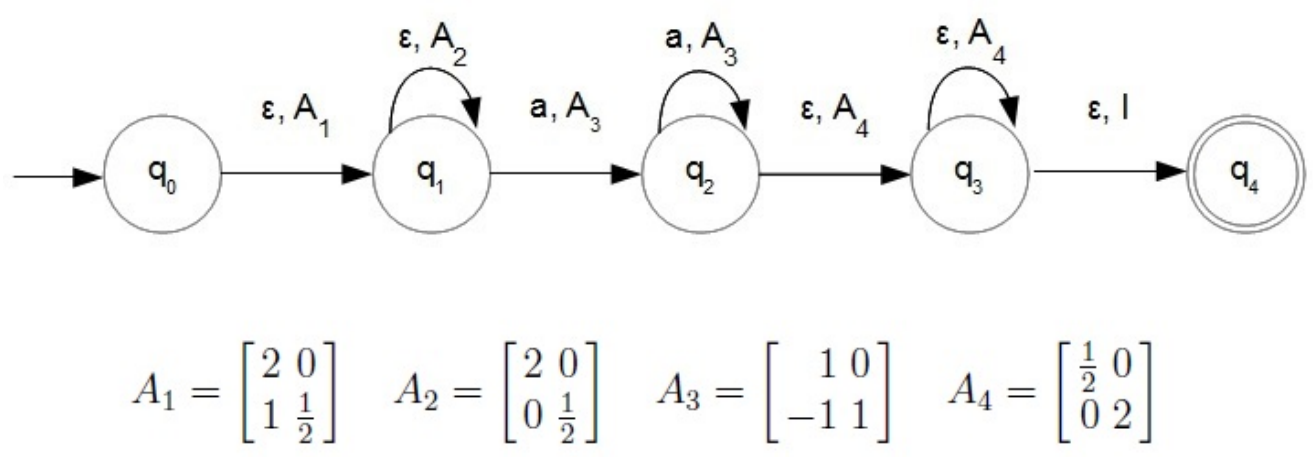

Figure 1: State diagram of $\mathcal{G}$ accepting the language $\mathrm{L}=\left\{a^{2^{2 n+1}} \mid n \geq 0\right\}$

as a result of the multiplications with the matrix $A_{1}$ and $x$ many $A_{2}$ 's. Multiplication with each $A_{3}$ leaves $2^{x+1}$ and $\frac{1}{2^{x+1}}$ unchanged while subtracting $\frac{1}{2^{x+1}}$ from $2^{x}$ for each scanned $a$. As a result of $y$ multiplications with $A_{3}$, the register will have the value

$$
\left[\begin{array}{cc}
2^{x+1} & 0 \\
2^{x}-\frac{y}{2^{x+1}} & \frac{1}{2^{x+1}}
\end{array}\right]
$$

For the rest of the computation, $\mathcal{G}$ will multiply its register with $A_{4}$ until nondeterministically moving to the final state. As a result of $z$ multiplications with $A_{4}$, the register will have the value

$$
\left[\begin{array}{cc}
\frac{2^{x+1}}{2^{z}} & 0 \\
\left(2^{x}-\frac{y}{2^{x+1}}\right) \frac{1}{2^{z}} & \frac{2^{z}}{2^{x+1}}
\end{array}\right] .
$$

The final value of the register is equal to the identity matrix when $y=2^{2 x+1}$ and $z=x+1$, which is possible only when the length of the input string is $2^{2 x+1}$ for some $x \geq 0$. In the successful branch, the register will be equal to the identity matrix and $\mathcal{G}$ will end up in the final state having successfully read the input string. For input strings which are not members of L, either the computation will end before reading the whole input string, or the final state will be reached with the register value not equaling the identity matrix.

Since the matrices used during the computation are 2 by 2 rational matrices with determinant $1, \mathrm{~L} \in \mathfrak{L}(S L(2, \mathbb{Q})) . \mathfrak{L}(S L(2, \mathbb{Q}))$ contains a unary nonregular language, which is not true for $\mathfrak{L}(S L(2, \mathbb{Z}))$ by Theorem 3.3 and we conclude the result.

We will now look at a special subgroup of $G L(2, \mathbb{Q})$.

For two integers $m$ and $n$, the Baumslag-Solitar group $B S(m, n)$ is defined as $B S(m, n)=$ $\left\langle a, b \mid b a^{m} b^{-1}=a^{n}\right\rangle$. We are going to focus on $B S(1,2)=\left\langle a, b \mid b a b^{-1}=a^{2}\right\rangle$.

Consider the matrix group $G_{B S}$ generated by the matrices 


$$
A=\left[\begin{array}{cc}
1 & 0 \\
-1 & 1
\end{array}\right] \quad \text { and } \quad B=\left[\begin{array}{cc}
1 / 2 & 0 \\
0 & 1
\end{array}\right]
$$

Consider the isomorphism $a \mapsto A, b \mapsto B$. The matrices $A$ and $B$ satisfy the property $B A B^{-1}=$ $A^{2}$

$$
\left[\begin{array}{cc}
1 / 2 & 0 \\
0 & 1
\end{array}\right]\left[\begin{array}{cc}
1 & 0 \\
-1 & 1
\end{array}\right]\left[\begin{array}{ll}
2 & 0 \\
0 & 1
\end{array}\right]=\left[\begin{array}{cc}
1 & 0 \\
-2 & 1
\end{array}\right],
$$

and we conclude that $G_{B S}$ is isomorphic to $B S(1,2)$.

We will prove that there exists a $B S(1,2)$-automaton which recognizes a non-context-free language.

Theorem $3.6 \mathfrak{L}(B S(1,2)) \nsubseteq \mathrm{CF}$.

Proof. Let us construct a $B S(1,2)$-automaton $\mathcal{G}$ recognizing the language UPOW $=\left\{a^{2^{n}} \mid n \geq 0\right\}$. The state diagram of $\mathcal{G}$ and the matrices are given in Figure 2. Without scanning any input symbol, $\mathcal{G}$ multiplies its register with the matrix $A_{1}$ successively. $\mathcal{G}$ nondeterministically moves to the next state reading the first input symbol without modifying the register. After that point, $\mathcal{G}$ starts reading the string and multiplies its register with the matrix $A_{2}$ for each scanned $a$. At some point, $\mathcal{G}$ nondeterministically stops reading the rest of the string and multiplies its register with the element $A_{3}$. After successive multiplications with $A_{3}, \mathcal{G}$ nondeterministically decides to move to an accept state.

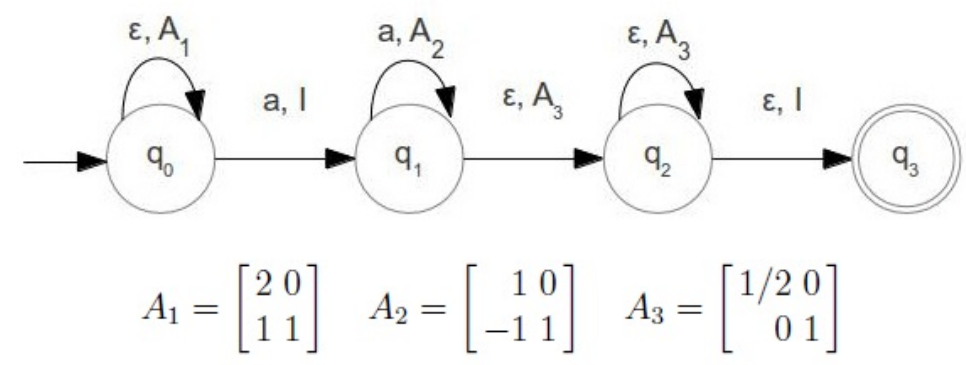

Figure 2: State diagram of $\mathcal{G}$ recognizing UPOW $=\left\{a^{2^{n}} \mid n \geq 0\right\}$

Before reading the first input symbol, the register has the value

$$
\left[\begin{array}{cc}
2^{k} & 0 \\
2^{k}-1 & 1
\end{array}\right]
$$

for some $k \geq 0$ as a result of the multiplications with the matrix $A_{1}$. Multiplication with each $A_{2}$ leaves $2^{k}$ unchanged while subtracting 1 from $2^{k}-1$ for each scanned $a$. For a member input string of the form $a^{2^{k}}$, in the successful branch $\mathcal{G}$ will multiply its register with $A_{2}$ until reaching the end of the string and the register will have the value

$$
\left[\begin{array}{cc}
2^{k} & 0 \\
0 & 1
\end{array}\right]
$$


For the rest of the computation, $\mathcal{G}$ will multiply its register with $A_{3}$ until nondeterministically moving to the final state. In the successful branch, the register will be equal to the identity matrix and $\mathcal{G}$ will end up in the final state having successfully read the input string. For input strings which are not members of UPOW, either the computation will end before reading the whole input string or the final state will be reached with the register value being different from the identity matrix. Note that $A_{1}=B^{-1} A^{-1}, A_{2}=A$ and $A_{3}=B$, where $A$ and $B$ are the generators of the group $G_{B S}$ and recall that $G_{B S}$ is isomorphic to $B S(1,2)$. Since UPOW is a unary nonregular language, it is not context-free and we conclude the result.

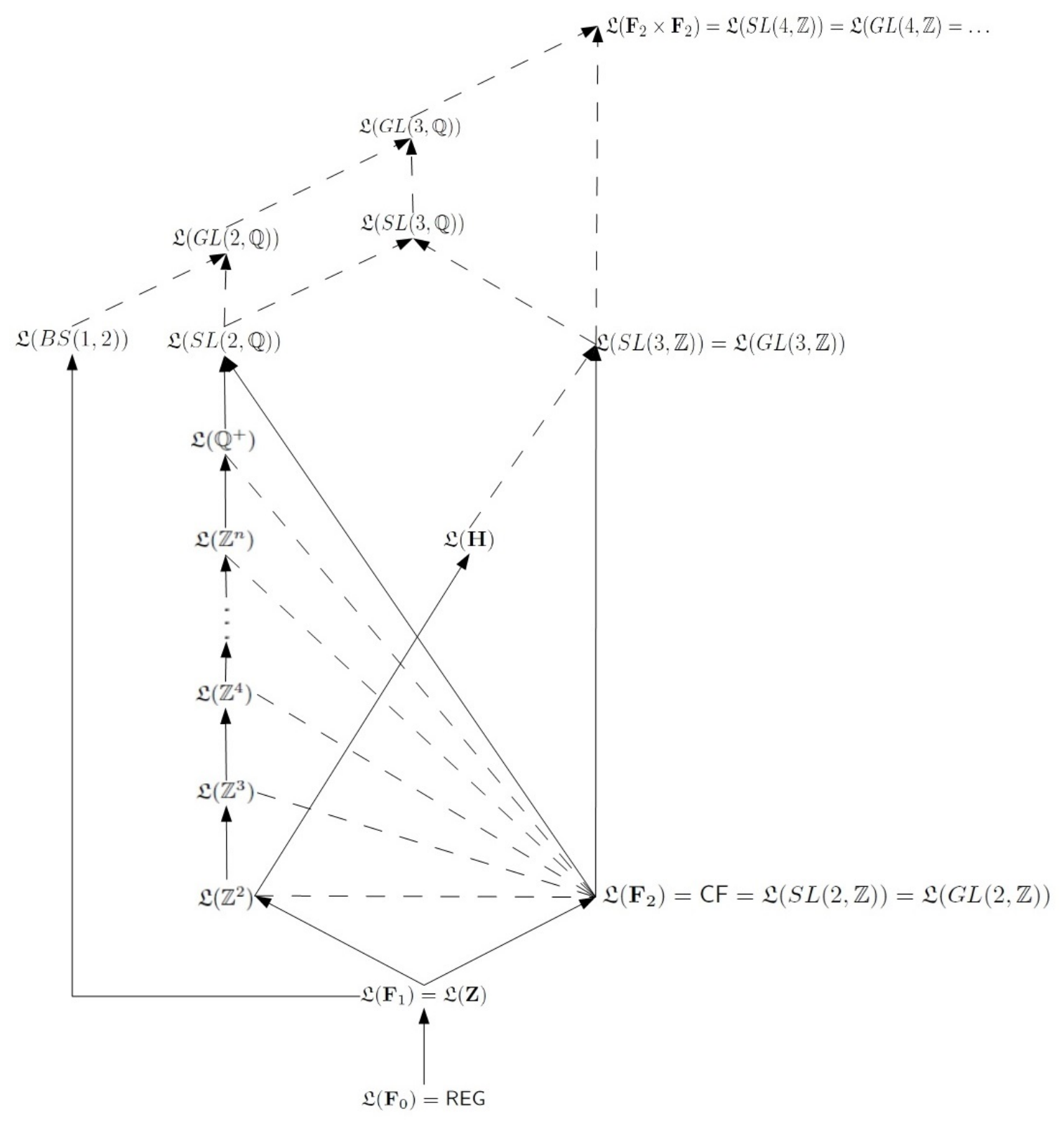

Figure 3: Language classes associated with groups

Note that $\mathfrak{L}(\mathbb{Z}) \subsetneq \mathfrak{L}(B S(1,2))$ since the subgroup generated by a in $B S(1,2)$ is isomorphic to 
$\mathbb{Z}$ and $\mathfrak{L}(B S(1,2))$ contains a unary nonregular language.

We summarize the results in Figure 3. Solid arrows represent proper inclusion, dashed arrows represent inclusion and dashed lines represent incomparability. For the relationships which are not discussed in this section, please see Section 5 .

\section{Time Complexity}

A group automaton $\mathcal{G}$ recognizing language $\mathrm{L}$ is said to operate in time $t(n)$, if for any input string $x$ with $|x|=n$ the computation of $\mathcal{G}$ takes at most $t(n)$ steps. We will denote the set of languages recognized by $G$-automata operating in time $t(n)$ by $\mathfrak{L}(G)_{t(n)}$.

Let $X$ be a generator set for $G$. The length of $g \in G$, denoted $|g|_{X}$, is the length of the shortest representative for $g$ in $X^{*}$. The growth function of a group $G$ with respect to a generating set $X$, denoted $g_{G}^{X}(n)$, is the cardinality of the set $\left\{g \in G,|g|_{X} \leq n\right\}$, that is the number of all elements in $G$ which can be represented by a word of length at most $n$. The growth function is asymptotically independent of the generating set, and we will denote the growth function of a group $G$ by $g_{G}(n)$.

For a positive integer $n$, two strings $w, w^{\prime} \in \Sigma^{*}$ are $n$-dissimilar, if $|w| \leq n,\left|w^{\prime}\right| \leq n$ and there exists a distinguishing string $v \in \Sigma^{*}$ with $|w v| \leq n,\left|w^{\prime} v\right| \leq n$ and $w v \in \mathrm{L}$ iff $w^{\prime} v \notin \mathrm{L}$. Let $N_{\mathrm{L}}(n)$ be the maximum $k$ such that there exist $k$ distinct strings that are pairwise $n$-dissimilar. For a string $w=w_{1} w_{2} \ldots w_{k} \in W(G), w^{-1}$ represents the word $w_{k}^{-1} w_{k-1}^{-1} \ldots w_{1}^{-1}$.

Lemma 4.1 Let $G$ be a finitely generated group with growth function $g_{G}(n) . \quad N_{W(G)}(n) \geq$ $g_{G}\left(\frac{n}{2}\right)$.

Proof. Let $X$ be the generator set of $G$. The strings in $W(G)$ are those which belong to $\left(X \cup X^{-1}\right)^{*}$ and represent the identity element of $G$. Let $w=w_{1} \ldots w_{k}$ be a word of length less than or equal to $\frac{n}{2}$. Each word of length $k$ can be extended with $w^{-1}=w_{k}^{-1} \ldots w_{1}^{-1}$ so that the extended word represents the identity element of $G$ and the word has length less than or equal to $n$. The number of distinct elements $g$ in $G$ which can be represented by a word of length less than or equal to $\frac{n}{2}$ is $g_{G}\left(\frac{n}{2}\right)$, which is the cardinality of the set $\left\{g \in G,|g|_{X} \leq \frac{n}{2}\right\}$. Since the set $\left\{g \in G,|g|_{X} \leq \frac{n}{2}\right\}$ contains only the shortest representative of each element, any two elements $g_{1}, g_{2}$ are distinct such that the string $g_{1} g_{1}^{-1} \in W(G)$ whereas the string $g_{2} g_{1}^{-1} \notin W(G)$. We conclude that there are at least $g_{G}\left(\frac{n}{2}\right)$ distinct strings that are pairwise $n$-dissimilar.

Theorem 4.2 Let $G$ and $H$ be groups with polynomial and exponential growth functions $g_{G}(n)$ and $g_{H}(n)$ respectively. $\mathfrak{L}(H) \nsubseteq \mathfrak{L}(G)_{t(n)}$ where $t(n)$ is a polynomial.

Proof. Let $\mathcal{G}$ be a $G$-automaton recognizing a language $L$ in time $t(n)$. A configuration of a group automaton consists of a state and a group element pair. Let us count the number of distinct configurations of $\mathcal{G}$ that can be reached after reading a string of length at most $m$. Since the number of states is constant, we will analyze the number of different group elements that can appear in the register. After reading a string of length exactly $m$, the product of the 
labels on the edges can be given by

$$
l=g_{i_{1}} g_{i_{2}} \ldots g_{i_{k}}
$$

for some $k \leq t(m)$. $l$ can be expressed as a product of $\kappa$ generators, where $\kappa$ is at most $C \cdot k$ for some constant $C$, since each group element is composed of at most some constant number of generators, which is independent of the length of the string. The number of elements in $G$ which can be represented as a product of at most $\kappa$ generators is given by $g_{G}(\kappa)$ by the definition of the growth function of $G$. Hence, the number of different values that can appear in the register after reading a string of length exactly $m$ is less than or equal to $g_{G}(\kappa)$. Since $\kappa \leq C \cdot k$ and $k \leq t(m)$, we can conclude that

$$
g_{G}(\kappa) \leq g_{G}(C \cdot t(m)) .
$$

$g_{G}(C \cdot t(m))$ is a polynomial function of $m$ since both the growth function of $G$ and $t(m)$ have polynomial growth. Now it is easy to see that the number of different group elements that can appear in the register after reading a string of length at most $m$, it is still a polynomial function of $m$.

Now let us consider the strings in $W(H) \cdot g_{H}\left(\frac{n}{2}\right) \leq N_{W(H)}(n)$ by Lemma 4.1. Hence, there are at least $g_{H}\left(\frac{n}{2}\right)$ many distinct $x_{i}, x_{j}$ such that $x_{i} y \in W(H)$ whereas $x_{j} y \notin W(H)$ and $\left|x_{i} y\right| \leq n$ and $\left|x_{j} y\right| \leq n$ for some $y$. Let us consider the accepting computation paths for these strings. After finishing reading the $x_{i}$, a configuration should be reached which eventually leads to an accept state. Since the total number of distinct configurations after reading a prefix of length at most $n$ is a polynomial function of $n$, the number of configurations which leads to an accept state is also a polynomial function of $n$. We can conclude that the same configuration should be reached after reading two distinct strings $x_{i}$ and $x_{j}$ since there are at least $g_{H}\left(\frac{n}{2}\right)$ many such different strings which is an exponential function of $n$. We have assumed that the configuration in consideration leads to an accept state, that is an accepting configuration is reached if the string $x_{i}$ is extended with $y$. This will result in the acceptance of the string $x_{j} y$, which is not a member of $W(H)$ since otherwise $x_{i}$ would be equivalent to $x_{j}$. We arrive at a contradiction and conclude that $W(H)$ cannot be recognized by a $G$-automaton in polynomial time.

We conclude that $\mathfrak{L}(H) \nsubseteq \mathfrak{L}(G)_{t(n)}$ since $W(H)$ is trivially in $\mathfrak{L}(H)$.

Theorem 4.3 Let $G$ be a group with a polynomial growth function. There exists a context-free language which cannot be recognized by any $G$-automaton in polynomial time.

Proof. It is known that the word problem of the free group of rank $W\left(\mathbf{F}_{2}\right)$ has an exponential growth function. Assuming that $G$ is a group with polynomial growth function, $W\left(\mathbf{F}_{2}\right)$ cannot be recognized by any $G$-automaton in polynomial time by Theorem 4.2 . Since $W\left(\mathbf{F}_{2}\right)$ is a context-free language, the proof is complete.

\section{Corollary 4.4 CF $\nsubseteq \mathfrak{L}(\mathbf{H})_{\text {Poly }}$.}

Proof. It is known that the Discrete Heisenberg group $\mathbf{H}$ has polynomial growth function. The result follows by Theorem 4.3 . 


\section{Additional Results}

In this section, we are going to state some known results from the literature and some new results which help us complete Figure 3 .

The relation between the classes of languages recognized by free group automata is summarized as follows.

Fact 5.15 REG $=\mathfrak{L}\left(\mathbf{F}_{0}\right) \subsetneq \mathfrak{L}\left(\mathbf{F}_{1}\right)=\mathfrak{L}(\mathbb{Z}) \subsetneq \mathfrak{L}\left(\mathbf{F}_{2}\right)$.

The following result states the hierarchy between the classes of languages recognized by $\mathbb{Z}^{n}$ automata. This result also follows from the hierarchy between the class of languages recognized by nondeterministic blind $k$-counter automata.

Fact $5.2 \mathfrak{L}\left(\mathbb{Z}^{k}\right) \subsetneq \mathfrak{L}\left(\mathbb{Z}^{k+1}\right)$ for $k \geq 1$.

At the top of the hierarchy of $\mathbb{Z}^{k}$, we place $\mathbb{Q}^{+}$, which is isomorphic to a free Abelian group of infinite rank. Let us note that the set of languages recognized by $\mathbb{Q}^{+}$-automata is a proper subset of the set of languages recognized by $S L(2, \mathbb{Q})$-automata which can be concluded with the help of the following fact.

Fact 5.3 [11] All 1NFAMW-recognizable languages over a unary alphabet are regular.

Theorem $5.4 \mathfrak{L}\left(\mathbb{Q}^{+}\right) \subsetneq \mathfrak{L}(S L(2, \mathbb{Q}))$.

Proof. Let $\mathrm{L} \in \mathfrak{L}\left(\mathbb{Q}^{+}\right)$and let $\mathcal{G}$ be a $\mathbb{Q}^{+}$-automaton recognizing L. We will construct a $\mathfrak{L}(S L(2, \mathbb{Q}))$-automaton $\mathcal{G}^{\prime}$ recognizing L. Let $S=\left\{s_{1}, \ldots, s_{n}\right\}$ be the set of elements multiplied with the register during the computation of $\mathcal{G}$. We define the mapping $\varphi$ as follows.

$$
\varphi: s_{i} \mapsto\left[\begin{array}{cc}
s_{i} & 0 \\
0 & \frac{1}{s_{i}}
\end{array}\right]
$$

The elements $\varphi\left(s_{i}\right)$ are $2 \times 2$ rational matrices with determinant 1 . Let $\delta$ and $\delta^{\prime}$ be the transition functions of $\mathcal{G}$ and $\mathcal{G}^{\prime}$ respectively. We let $\left(q^{\prime}, s_{i}\right) \in \delta(q, \sigma) \Longleftrightarrow\left(q^{\prime}, \varphi\left(s_{i}\right)\right) \in \delta^{\prime}(q, \sigma)$ for every $q, q^{\prime} \in Q, \sigma \in \Sigma$ and $s_{i} \in S$. The resulting $\mathcal{G}^{\prime}$ recognizes L.

The inclusion is proper since $\mathrm{L}=\left\{a^{2^{2 n+1}} \mid n \geq 0\right\} \in \mathfrak{L}(S L(2, \mathbb{Q}))$ by Theorem 3.5 , and $\mathfrak{L}\left(\mathbb{Q}^{+}\right)$ does not contain any unary nonregular languages by Fact 5.3 , noting that $\mathbb{Q}^{+}$-automata are equivalent to 1 NFAMW's.

Let us mention that the class of context-free languages and the class of languages recognized by nondeterministic blind counter automata are incomparable.

Fact 5.5 CF and $\mathfrak{L}\left(\mathbb{Z}^{n}\right)$ are incomparable for all $n \geq 2$.

Proof. Consider the language $\mathrm{L}=\left\{a^{n} b^{n} \mid n \geq 0\right\}$ which is a context-free language. Since context-free languages are closed under star, $\mathrm{L}^{*}$ is a context-free language whereas it cannot be 
recognized by any $\mathbb{Z}^{n}$-automaton for all $n \geq 1$ by [10]. On the other hand, the non-context-free language $\mathrm{L}^{\prime}=\left\{a^{n} b^{n} c^{n} \mid n \geq 0\right\}$ can be recognized by a $\mathbb{Z}^{2}$-automaton.

Let us move on to the results about linear groups. The following result is a direct consequence of Fact 3.2 .

Theorem 5.6 $\mathfrak{L}(S L(3, \mathbb{Z}))=\mathfrak{L}(G L(3, \mathbb{Z}))$.

Proof. Since $G L(3, \mathbb{Z})$ is a finitely generated group and $S L(3, \mathbb{Z})$ has finite index in $G L(3, \mathbb{Z})$, the result follows by Fact 3.2 .

We have talked about the discrete Heisenberg group $\mathbf{H}$, an important subgroup of $S L(3, \mathbb{Z})$. Now let us look at a subgroup of $\mathbf{H}$ generated by the matrices $B$ and $C$ which we will call $\mathbf{G}_{\mathbf{2}}$.

$$
B=\left[\begin{array}{lll}
1 & 0 & 0 \\
0 & 1 & 1 \\
0 & 0 & 1
\end{array}\right] \quad C=\left[\begin{array}{lll}
1 & 0 & 1 \\
0 & 1 & 0 \\
0 & 0 & 1
\end{array}\right]
$$

$\mathbf{G}_{\mathbf{2}}=\langle B, C \mid B C=C B\rangle$ is a free Abelian group of rank 2 and therefore it is isomorphic to $\mathbb{Z}^{2}$.

We conclude the following about the language recognition power of $\mathbb{Z}^{2}$ and $\mathbf{H}$.

Theorem $5.7 \mathfrak{L}\left(\mathbb{Z}^{2}\right) \subsetneq \mathfrak{L}(\mathbf{H})$.

Proof. Since $\mathbb{Z}^{2}$ is a subgroup of $\mathbf{H}, \mathfrak{L}\left(\mathbb{Z}^{2}\right) \subseteq \mathfrak{L}(\mathbf{H})$ follows. The inclusion is proper since $\mathbf{H}$ can recognize the unary nonregular language COMPOSITE $=\left\{x^{p q} \mid p, q>1\right\}$ by [18], which is not possible for any $\mathbb{Z}^{n}$-automaton by Fact 5.3 .

In [17], it is proven that $\mathbf{F}_{2} \times \mathbf{F}_{2}$-automaton is as powerful as a Turing machine, which places $\mathbf{F}_{2} \times \mathbf{F}_{2}$ at the top of the language hierarchy of group automata.

Fact $5.8\left[17 \mathfrak{L}\left(\mathbf{F}_{2} \times \mathbf{F}_{2}\right)\right.$ is the family of recursively enumerable languages.

We make the following observation.

Theorem 5.9 RE $=\mathfrak{L}\left(\mathbf{F}_{2} \times \mathbf{F}_{2}\right)=\mathfrak{L}(S L(n, \mathbb{Z}))$ for $n \geq 4$.

Proof. The first equality is Fact 5.8. Recall that $\varphi$ is an isomorphism from $\mathbf{F}_{2}$ onto $\mathbf{G}$, the matrix group generated by the matrices $M_{a}$ and $M_{b}$. Let $\mathbf{G}^{\prime}$ be the following group of matrices

$$
\left\{\left[\begin{array}{ccc}
M_{1} & 0 & 0 \\
0 & 0 & 0 \\
0 & 0 &
\end{array}\right], M_{2}, M_{2} \in \mathbf{G}\right\} .
$$

We will define the mapping $\psi: \mathbf{F}_{2} \times \mathbf{F}_{2} \rightarrow \mathbf{G}^{\prime}$ as $\psi\left(g_{1}, g_{2}\right)=\left(\varphi\left(g_{1}\right), \varphi\left(g_{2}\right)\right)$ for all $\left(g_{1}, g_{2}\right) \in$ $\mathbf{F}_{2} \times \mathbf{F}_{2}$ which is an isomorphism from $\mathbf{F}_{2} \times \mathbf{F}_{2}$ onto $\mathbf{G}^{\prime}$. 
This proves that $\mathbf{F}_{2} \times \mathbf{F}_{2}$ is isomorphic to a subgroup of $S L(4, \mathbb{Z})$. The fact that $\mathfrak{L}\left(\mathbf{F}_{2} \times \mathbf{F}_{2}\right)$ is the set of recursively enumerable languages helps us to conclude that $\mathfrak{L}(S L(n, \mathbb{Z}))$ is the set of recursively enumerable languages for $n \geq 4$.

Let us also state that the classes of languages recognized by automata over supergroups of $S L(4, \mathbb{Z})$ such as $G L(4, \mathbb{Z})$ or $S L(4, \mathbb{Q})$ are also identical to the class of recursively enumerable languages, since such automata can be simulated by Turing machines.

\section{Open Questions}

Does there exist a $S L(3, \mathbb{Z})$-automaton recognizing $W\left(\mathbb{Z}^{3}\right)$ ? ${ }^{1}$

Can we prove a stronger version of Theorem 4.3 , which is independent of the time component? For instance, for the case of $\mathbf{F}_{2}$, is it true that $W\left(\mathbf{F}_{2}\right) \notin \mathfrak{L}(\mathbf{H})$ in general?

Can we describe the necessary properties of a group $G$ so that $\mathfrak{L}(G)$ contains $W\left(\mathbf{F}_{2}\right)$ ?

Little is known about $B S(1,2)$-automata. Does $\mathfrak{L}(B S(1,2))$ contain every context-free language?

Which, if any, of the subset relationships in Figure 3 are proper inclusions?

\section{Acknowledgements}

We thank the anonymous reviewers for their constructive comments.

\section{References}

[1] G. BAUMSLAG, J. E. ROSEBLADE, Subgroups of direct products of free groups. Journal of the London Mathematical Society 2 (1984) 1, 44-52.

[2] N. P. BROWN, N. OZAWA, $C^{*}$-algebras and finite-dimensional approximations. 88, American Mathematical Soc., 2008.

\footnotetext{
${ }^{1}$ Corollary 2 of $[3]$ states that the word problem of a finitely generated Abelian group $H$ is recognized by a $G$-automaton if and only if $H$ has a finite index subgroup isomorphic to a subgroup of $G$. That corollary could be used to give an affirmative answer to this open question. Unfortunately, the corollary is wrong: Let $H$ be an Abelian group and let $G=\mathbf{F}_{2} \times \mathbf{F}_{2} . \mathfrak{L}\left(\mathbf{F}_{2} \times \mathbf{F}_{2}\right)$ contains the word problem of any finitely generated Abelian group. Since $\mathbf{F}_{2} \times \mathbf{F}_{2}$ is finitely generated, any finite index subgroup of $\mathbf{F}_{2} \times \mathbf{F}_{2}$ is also finitely generated. Any finite index subgroup of $\mathbf{F}_{2} \times \mathbf{F}_{2}$ is either free or has a subgroup of finite index that is a direct product of free groups [1. Any subgroup of an Abelian group is again Abelian. Hence, it is not possible that $G$ has a finite index subgroup isomorphic to a subgroup of $H$.
} 
[3] S. CLEARY, M. ELDER, G. OSTHEIMER, The word problem distinguishes counter languages. arXiv preprint math/0606415 (2006).

[4] J. M. CORSON, Extended finite automata and word problems. International Journal of Algebra and Computation 15 (2005) 03, 455-466.

[5] J. DASSOW, V. MITRANA, Finite automata over free groups. International Journal of Algebra and Computation 10 (2000) 06, 725-737.

[6] M. ELDER, M. KAMBITES, G. OSTHEIMER, On groups and counter automata. International Journal of Algebra and Computation 18 (2008) 08, 1345-1364.

[7] G. Z. ELSTON, G. OSTHEIMER, On groups whose word problem is solved by a counter automaton. Theoretical Computer Science 320 (2004) 23, 175 - 185.

[8] P. C. FISCHER, A. R. MEYER, A. L. ROSENBERG, Real time counter machines. In: Proceedings of the 8th Annual Symposium on Switching and Automata Theory (SWAT 1967). FOCS '67, 1967, 148-154.

[9] J. FRALEIGH, V. KATZ, A first course in abstract algebra. Addison-Wesley world student series, Addison-Wesley, 2003.

[10] S. A. GREIBACH, Remarks on blind and partially blind one-way multicounter machines. Theoretical Computer Science 7 (1978), 311-324.

[11] O. H. IBARRA, S. K. SAHNI, C. E. KIM, Finite automata with multiplication. Theoretical Computer Science 2 (1976) 3, 271 - 294.

[12] M. KAMBITES, Word problems recognisable by deterministic blind monoid automata. Theor. Comput. Sci. 362 (2006) 1, 232-237.

[13] M. KAMBITES, Formal languages and groups as memory. Communications in Algebra 37 (2009) 1, 193-208.

[14] M. I. KARGAPOLOV, J. I. MERZLJAKOV, Fundamentals of the Theory of Groups. SpringerVerlag, 1979.

[15] R. C. LYNDON, P. E. SCHUPP, Combinatorial Group Theory. Springer-Verlag, 1977.

[16] V. MitRAnA, R. STIEBE, The Accepting Power of Finite Automata over Groups. In: New Trends in Formal Languages. Springer-Verlag, 1997, 39-48.

[17] V. MITRANA, R. STIEBE, Extended finite automata over groups. Discrete Appl. Math. 108 (2001) 3, 287-300.

[18] E. RENDER, Rational monoid and semigroup automata. Ph.D. thesis, University of Manchester, 2010 . 